

\title{
A theoretical study of shock front propagation in the density graded cellular rods
}

Jiagui Liu, Bing Hou, Fangyun Lu, Han Zhao

\section{To cite this version:}

Jiagui Liu, Bing Hou, Fangyun Lu, Han Zhao. A theoretical study of shock front propagation in the density graded cellular rods. International Journal of Impact Engineering, 2015, 80, pp.133-142. 10.1016/j.ijimpeng.2015.02.001 . hal-01137279

\section{HAL Id: hal-01137279 \\ https://hal.science/hal-01137279}

Submitted on 30 Mar 2015

HAL is a multi-disciplinary open access archive for the deposit and dissemination of scientific research documents, whether they are published or not. The documents may come from teaching and research institutions in France or abroad, or from public or private research centers.
L'archive ouverte pluridisciplinaire HAL, est destinée au dépôt et à la diffusion de documents scientifiques de niveau recherche, publiés ou non, émanant des établissements d'enseignement et de recherche français ou étrangers, des laboratoires publics ou privés. 


\title{
A theoretical study of shock front propagation in the density graded cellular rods
}

\author{
Jiagui Liu ${ }^{\mathrm{a}, \mathrm{b}}$, Bing Hou ${ }^{\mathrm{c}}$, Fangyun Lu ${ }^{\mathrm{d}}$, Han Zhao ${ }^{\mathrm{b}, *}$ \\ a State Key Laboratory for Disaster Prevention and Mitigation of Explosion and Impact, PLA University of \\ Science and Technology, 210007, Nanjing, China \\ ${ }^{b}$ Laboratoire de Mécanique et Technologie (LMT-Cachan), ENS-Cachan/CNRS-UMR8535/Université Pierre E \\ Marie Curie, 61, avenue du président Wilson, 94235 Cachan cedex, France \\ ${ }^{c}$ School of Aeronautics, Northwestern Polytechnical University, 710072 Xi'an, China \\ ${ }^{d}$ Institute of Technique Physics, College of Science, National University of Defense Technology, 410073, \\ Changsha, China
}

\begin{abstract}
The present theoretical study focuses on the shock front propagation in a density graded cellular rod. It aims at the determination of the shock front velocity evolution as well as the stress evolution during the impact. The density gradient leads necessarily to the property gradient, which is taken into account in this analysis. The locking strain in classical shock front theory is replaced by the notion of the locking density to adapt to the studied case. Analytical solutions of linear, quadratic and square root density profiles are obtained. They are compared to the FEM solution with a good agreement. This study reveals a possibility to reduce the maximum impact stress for the impacted structures (same impacting mass, same energy absorption) using a proper density gradient.
\end{abstract}

Keywords: Shock front propagation, Cellular materials, Density graded cellular rod, Energy absorption

\section{Introduction}

Cellular materials (e.g. honeycomb, stochastic foam, hollow sphere agglomerate and lattice truss core material) have attracted important interests in both fundamental researches and industrial applications due to their high specific strength and good energy absorption capabilities. Therefore, a great number of experimental, numerical and analytical studies on the behaviour of cellular materials under quasi-static and dynamic loading have been reported in the past decades, showing that (i) the behaviour of a cellular material can be derived from its base material (cell

\footnotetext{
*Corresponding author zhao@lmt.ens-cachan.fr.
} 
wall material) and the relative density with a reasonable accuracy (Gibson and Ashby, 1997); (ii) their strain rate sensitivities come not only from the base material rate sensitivity but also from structural effects (Zhao et al., 2005; Hou et al., 2012).

Among those researches, an interesting feature is the shock front formation within the cellular materials under high speed impact. The pioneer study in the domain was carried out by Reid and Peng (1997). They observed a huge strength increase when the balsa wood specimens were shoot at a velocity up to $250 \mathrm{~m} / \mathrm{s}$ against an instrumented Hopkinson bar. In order to explain the measured increases which seemed to be proportional to the square of the impact velocity, they developed a rigid perfectly plastic locking (RPPL) shock model.

The basic assumption of this model is that there exists a moving shock front separating the specimen into two zones: one (ahead of shock front) is rigid perfectly plastic and the other (behind the front) is compacted to a constant locking strain $\varepsilon_{d}$.

The conservation laws lead to the following estimate of the stress behind the shock front $\sigma_{d}$, called afterward dynamic crushing stress:

$$
\sigma_{d}=\sigma_{p}+\frac{\rho_{0}}{\varepsilon_{d}} V^{2}
$$

This one-dimensional shock theory framework was successfully applied by a number of previous works to various metallic foams (Tan et al., 2005; Hanssen et al., 2002; Radford et al., 2005; Tan et al., 2012). Experimental observations of those two distinct zones with high speed camera (Elnasri et al., 2007; Nemat-Nasser et al., 2007) gave a physical proof.

Improvements of such a theory were also reported. Lopatnikov et al. (2003, 2004) proposed to take into account the initial elasticity with an elastic perfectly plastic rigid model (EPPR). Pattofatto et al. (2007) used a power law shock model to reduce the effect of a constant locking strain. Zheng et al. (2012) proposed another extension of RPPL model using a linearly hardening plastic locking model (R-LHP-L).

Recently, the idea of functionally graded cellular materials was spread out. Gupta (2007) investigated the hollow particles filled by functionally graded syntactic foam under quasi-static loading, in which the gradient was achieved by agglomerating different densities of hollow particles. Cui et al. (2009) extensively studied the influence in energy absorption capability of foam with five different density gradient profiles under low speed impact loading and concluded that functionally graded foams were better in energy absorption than the uniform foam. Honeycomblike structures with gradient were also theoretically and numerically investigated (Ali et al., 2008; Ajdari et al., 2011). Zeng et al. (2010) investigated the influence of the density gradient 
profile on the mechanical properties of the graded polymeric hollow sphere agglomerates with Hopkinson bars techniques and direct impact tests. The experimental results showed that the gradient profile played an important role in both energy absorption and the transmitted force. Wang et al. (2013) investigate the energy absorption capacity of density graded Voronoi honeycomb with a numerical method. Another kind of gradient, varying cross-section, can also be found (Shen et al., 2013b).

With a property gradient, a very interesting question is the influence of the gradient on the shock front formation/propagation. Indeed, Shen et al. (2013a) presented an analytical study of the shock front propagation in property graded cellular rods. They proposed that the plastic plateau stress of the cellular material varied with its position within the cellular rod while the initial relative density remained the constant. This assumption permits to apply easily the framework of RPPL model proposed by Reid and Peng (1997) with a constant locking strain. Results showed a significant influence of property gradient.

However, from the theoretical analysis provided by Gibson and Ashbv (1997), the quasi-static yield stress $\sigma_{p}$ should be expressed as

$$
\sigma_{p}=B\left(\frac{\rho_{0}}{\rho_{s}}\right)^{3 / 2} \sigma_{y s}
$$

where $\rho_{0}$ and $\rho_{s}$ are respectively the density of the foam and that of base material, $\sigma_{y s}$ denotes the yielding stress of the base material and $B$ a constant determined by experimental data.

Thus, the gradient introduced by Shen et al. (2013a) was issued from an assumption where gradient was achieved by the yield stress variation of the base material $\sigma_{y s}$ without any density change. However, in the industrial manufacture, the property gradient is generally obtained with the density gradients (Gupta, 2007; Zeng et al., 2010; Hohe et al., 2012).

This paper tends to study the shock front formation/propagation in a cellular rod with property gradient due to relative density gradient. The main difficulty lies in the fact that the locking strain can not be constant so that the basic framework RPPL model in the previous literature can not be applied anymore. The basic assumption of this work is that the locking will take place at a given density. Indeed, the locking strain for cellular structures made of same base material and similar morphology varies with its initial porosities. However, at this locking point, the density of all those cellular material (same base material and morphology) is more or less the same. For example, if densification is idealised as a no porosity state, cellular structure made of same base materials will densify at the same base material density.

This assumption leads to an extension of the shock front theory to the case of the density 
gradient cellular rods. The theoretical analysis is presented and analytical solutions are found for linear, quadratic and square root gradient profiles. Finally, the effect of density gradient on the shock front formation/propagation in terms of resulting shock stress as well as energy absorption capacity are discussed in the cases of cellular rod with and without a backing mass.

\section{Shock front in a cellular rod with density gradient}

\subsection{From locking strain to locking density}

As mentioned in the introduction, the theory of shock formation in cellular materials is built with the notion of locking strain where cellular material begins to densify. This locking strain varies with the initial density of the cellular material and it is difficult to apply this notion for a cellular rod with a density gradient. However, this locking strain denotes the state where nearly all the initial voids have been compressed. It means also that at this state the density of the compressed cellular materials should have a given density (or given ratio between its density and the density of base materials). This idea leads to the notion of the locking density which is the starting point of our analysis.

The introduction of locking density is based on the theoretical prediction of densification strain:

$$
\varepsilon_{d}=1-D \frac{\rho_{0}}{\rho_{s}}
$$

This is a first order simplification, more accurate prediction should include an item of the third order of the relative density $\left(\rho_{0} / \rho_{s}\right)($ Ashbv et al., 2000). Actually, the first order form is widely used in literatures for simplicity. Gibson and Ashby (1997) suggested a value of 1.4 for the parameter $D$ while $\mathrm{Hu}$ and $\mathrm{Yu}(2010)$ deduced the value of $D(=4 / 3)$ by a theoretical analysis of honeycomb crush and this was also validated by FEM simulation. Based on these works, we can transform the Eq. (3) as

$$
\varepsilon_{d}=1-\frac{\rho_{0}}{\rho_{s} / D}
$$

Thus, locking density $\left(\rho_{d}\right)$ is introduced as following:

$$
\rho_{d}=\frac{\rho_{s}}{D}
$$

Therefore, the locking strain reads as Eq. (6).

$$
\varepsilon_{d}=1-\frac{\rho_{0}}{\rho_{d}}
$$


where $\rho_{0}$ and $\rho_{d}$ are the densities ahead of and behind shock front.

In order to verify this assumption, the ratio between the density at the locking strain and the base material density is calculated for the random cellular structures of various initial density (polymeric/aluminum foam, hollow sphere agglomerates, etc) available in open literature. All the experimental data leads to a more or less constant value. It manifests therefore that the locking density is rather independent to the initial density of cellular materials. Such an experimental observation is not exhaustive and it is only valid for listed materials and in the aformentioned range of impact velocity.

It was reported by the previous works of Tan et al. (2012) and Zou et al. (2009) that the locking strain is inevitably dependent on the impact velocity. Here, the data collected contains experimental results under quasi-static as well as moderate impact loading $(<100 \mathrm{~m} / \mathrm{s})$. The case of extremely high speed plate impact testing (around $\mathrm{km} / \mathrm{s}$ ) are not considered in this work.

It is also emphasized that our study is limited in the case that the density gradient is rather small and the highest relative density of graded cellular bar is also small. With those limitations, the cell morphology variation and other induced structure effect due to density gradient can be neglected.

Therefore, in this studied density graded cellular rod, the locking density $\rho_{d}$ is considered as a constant. However, the locking strain varies with the initial density of cellular materials, which depends on the current spatial coordinate.

\subsection{Governing equations}

Considering a cellular rod launched against the rigid wall with an initial velocity $V_{0}$ as shown in Fig. 1, the initial length of the rod is $l_{0}$ and the density of the $\operatorname{rod} \rho(Z)$ is a function of its initial Lagrangian coordinate $Z$.

At the instant $t$ after the impact, it is assumed that there exists a shock front propagating along the rod behind which the density is $\rho_{d}$ and the particle velocity zero. Assuming that this front arrives at $x$ and the rear free end of the rod moves a distance of $u$, the position of shock front in Lagrangian coordinate $Z$ is expressed from the geometrical relationship (see Fig. 1) as follows:

$$
Z=x+u
$$

The mass conservation law applied to portion of compacted rod (behind shock front) gives: 


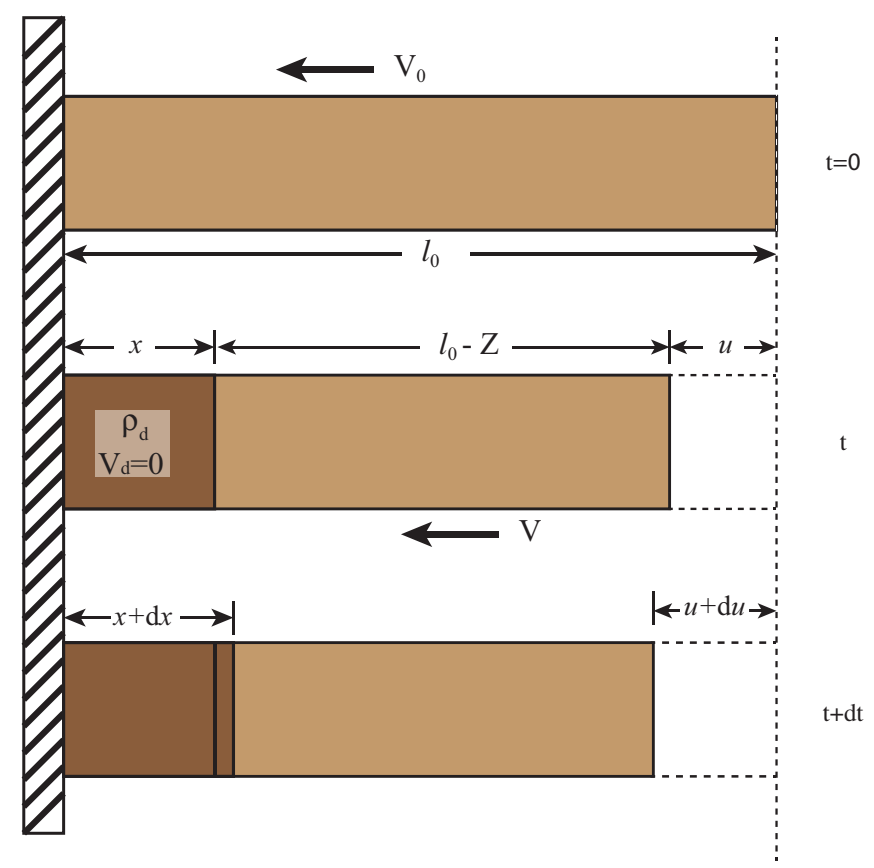

Figure 1: The diagram of direct impact experiment against a rigid wall

$$
\rho_{d} A_{0} x=m=\int_{0}^{Z} \rho(l) A_{0} \mathrm{~d} l
$$

Where $A_{0}$ is the cross section area of rod and $m$ the mass of compacted portion. Then,

$$
x=\frac{\int_{0}^{Z} \rho(l) \mathrm{d} l}{\rho_{d}}
$$

Differentiating Eq. (8) and Eq. (9), we get separately

$$
\begin{array}{r}
\frac{\mathrm{d} m}{\mathrm{~d} t}=\rho(Z) A_{0} \frac{\mathrm{d} Z}{\mathrm{~d} t} \\
\frac{\mathrm{d} x}{\mathrm{~d} t}=\frac{\rho(Z)}{\rho_{d}} \frac{\mathrm{d} Z}{\mathrm{~d} t}
\end{array}
$$

Substituting Eq. (11) into the differential form of Eq. (7), we get the expression of $V$, the velocity of the free end:

$$
V=\frac{\mathrm{d} u}{\mathrm{~d} t}=\frac{\mathrm{d} Z}{\mathrm{~d} t}-\frac{\mathrm{d} x}{\mathrm{~d} t}=\left[1-\frac{\rho(Z)}{\rho_{d}}\right] \frac{\mathrm{d} Z}{\mathrm{~d} t}
$$

Besides, the conservation of the momentum of the portion ahead of shock front gives:

$$
-\sigma_{p}(Z) A_{0} \mathrm{~d} t=(M-m) \mathrm{d} V
$$

Here, $M$ denotes the total mass of the cellular rod and plastic plateau stress, $\sigma_{p}(Z)$, can be known from Eq. (2) by replacing the constant density of foam $\rho_{0}$ by $\rho(Z)$ : 


$$
\sigma_{p}(Z)=B\left[\frac{\rho(Z)}{\rho_{s}}\right]^{3 / 2} \sigma_{y s}
$$

By substituting the expression of $V$ given in Eq. (12), the momentum conservation equation, Eq. (13), becomes

$$
\sigma_{p}(Z) A_{0}=-(M-m) \frac{\mathrm{d}}{\mathrm{d} t}\left\{\left[1-\frac{\rho(Z)}{\rho_{d}}\right] \frac{\mathrm{d} Z}{\mathrm{~d} t}\right\}
$$

Finally, for a given density gradient profile $\rho(Z)$, Eq. (15) totally defines a differential equation of Lagrangian coordinate $Z$, which is the exact position of shock front.

Knowing that the particle velocity of the mass behind the shock front is zero, the dynamic crushing stress $\sigma_{d}$ (spatially constant in the compacted portion) can be evaluated by the momentum conservation of the entire system.

$$
-\sigma_{d} A_{0} \mathrm{~d} t=\mathrm{d}[(M-m) V]
$$

By substituting Eqs. (10, 12 and 13) into the above equation, we get

$$
\begin{aligned}
\sigma_{d} & =\frac{V}{A_{0}} \frac{\mathrm{d} m}{\mathrm{~d} t}-(M-m) \frac{\mathrm{d} V}{\mathrm{~d} t} \\
& =\rho(Z)\left[1-\frac{\rho(Z)}{\rho_{d}}\right]\left(\frac{\mathrm{d} Z}{\mathrm{~d} t}\right)^{2}+\sigma_{p}(Z)
\end{aligned}
$$

The derived governing equation of shock front in the graded cellular materials is quite general without conditions on the gradient profile. In particular, if the density function $\rho(Z)$ is replaced by constant density $\rho_{0}$, the locking strain will be derived with the Eq. (9) and it equals well to those given in the classical RPPL model (Reid and Peng, 1997; Harrigan et al., 2010).

$$
\varepsilon_{d}=\frac{Z-x}{Z}=1-\frac{\rho_{0}}{\rho_{d}}
$$

In the same way, the expression of dynamic crushing stress $\sigma_{d}$ in this case will also be reduced to the classic expression in Eq. (1).

In order further simplify the expression, the non-dimensional parameters can be introduced. Indeed, the Lagrangien coordinate becomes $\zeta=Z / l_{0}$. By introducing $\bar{\rho}(\zeta)$ defined as $\rho(\zeta) / \rho_{0}$ for brevity hereinafter, the plastic plateau stress is then expressed as

$$
\sigma_{p}(\zeta)=C \bar{\rho}(\zeta)^{3 / 2} \sigma_{y s}
$$

where $C=B\left(\frac{\rho_{0}}{\rho_{s}}\right)^{3 / 2}$. We can also define following non-dimensional parameters :

$$
\alpha=\rho_{0} / \rho_{d}, \quad \tau=C_{0} t / l_{0}
$$


Where $C_{0}$ equals to $\sqrt{\sigma_{y s} / \rho_{0}}$. Thus, the governing equation, Eq. (15), is rewritten in a nondimensional way:

$$
C \bar{\rho}(\zeta)^{3 / 2}=-[1-\bar{m}(\zeta)] \frac{\mathrm{d}}{\mathrm{d} \tau}\left\{\frac{\mathrm{d} \zeta}{\mathrm{d} \tau}[1-\alpha \bar{\rho}(\zeta)]\right\}
$$

where $\bar{m}(\zeta)$ is

$$
\bar{m}(\zeta)=\int_{0}^{\zeta} \bar{\rho}(l) \mathrm{d} l
$$

Meanwhile, the dynamic crushing stress defined in Eq. (17) becomes

$$
\frac{\sigma_{d}}{\sigma_{y s}}=\bar{\rho}(\zeta)[1-\alpha \bar{\rho}(\zeta)]\left(\frac{\mathrm{d} \zeta}{\mathrm{d} \tau}\right)^{2}+C \bar{\rho}(\zeta)^{3 / 2}
$$

\subsection{Analytical solutions for specific density gradient profiles}

The non-dimensional governing equation of shock front, Eq. (21), can be analytically solved for several specific density gradient profiles. The cases of uniform, linear, quadratic and square root, are investigated in the present study.

Assuming that the density profile is a combination of uniform, linear, quadratic and square root functions, it leads to the following expression:

$$
\bar{\rho}(\zeta)=\frac{1}{A}\left(1+a_{1} \zeta+a_{2} \zeta^{2}+a_{3} \sqrt{\zeta}\right)
$$

In order to keep the total mass of the rod unchanged and equal to $\rho_{0} A_{0} l_{0}$, the parameter $A$ should be set to:

$$
A=1+\frac{a_{1}}{2}+\frac{a_{2}}{3}+\frac{2 a_{3}}{3}
$$

Each specific density gradient profile can be obtained just by setting several parameters $a_{i}$ equal to 0 . The four aforementioned density profiles are shown in Eq. (26), and we can solve corresponding form of governing equation (Eq. (21) ) case by case.

$$
\bar{\rho}(\zeta)=\frac{\rho(\zeta)}{\rho_{0}}=\left\{\begin{array}{cccl}
1, & a_{1}=a_{2}=a_{3}=0, & \text { Uniform } \\
\frac{1+a_{1} \zeta}{1+1 / 2 a_{1}}, & a_{1} \neq 0 \quad a_{2}=a_{3}=0, & \text { Linear } \\
\frac{1+a_{2} \zeta^{2}}{1+1 / 3 a_{2}}, & a_{2} \neq 0 \quad a_{1}=a_{3}=0, & \text { Quadratic } \\
\frac{1+a_{3} \sqrt{\zeta}}{1+2 / 3 a_{3}}, & a_{3} \neq 0 \quad a_{1}=a_{2}=0, & \text { Square root. }
\end{array}\right.
$$




\subsubsection{Uniform density}

With a uniform rod, $a_{1}, a_{2}$ and $a_{2}$ are equal to 0. Eq. (21) is therefore simplified as follows:

$$
\frac{C}{1-\alpha}=-(1-\zeta) \frac{\mathrm{d}^{2} \zeta}{\mathrm{d} \tau^{2}}
$$

The general solution of the differential equation above is:

$$
\left(\frac{\mathrm{d} \zeta}{\mathrm{d} \tau}\right)^{2}=\frac{2 C}{1-\alpha} \ln (1-\zeta)+\text { Const }
$$

The integral constant can be identified with the initial condition, that is:

$$
\left.\frac{\mathrm{d} \zeta}{\mathrm{d} \tau}\right|_{\tau=0}=\frac{\bar{V}}{1-\alpha}
$$

where $\bar{V}$ is non-dimensional value, $V_{0} / C_{0}$. The solution for uniform density rod is thus:

$$
\frac{\mathrm{d} \zeta}{\mathrm{d} \tau}=\frac{\bar{V}}{1-\alpha} \sqrt{1+\frac{2 C(1-\alpha)}{\bar{V}^{2}} \ln (1-\zeta)}
$$

This expression of $\frac{\mathrm{d} \zeta}{\mathrm{d} \tau}$ describes the shock front speed when it arrives at the position $\zeta$. It is noted that the same result is found as previous work by Lopatnikov et al. (2003).

The dynamic crushing stress when the shock front arrives at the position $\zeta$ is thus computed by Eq. (23),

$$
\frac{\sigma_{d}}{\sigma_{y s}}=\frac{\bar{V}^{2}}{1-\alpha}+2 C \ln (1-\zeta)+C
$$

\subsubsection{Linear density}

For the linear density gradient rod, $a_{2}$ and $a_{3}$ are equal to 0 . Eq. (21) is therefore transformed to:

and then:

$$
C\left[\frac{1}{A}\left(1+a_{1} \zeta\right)\right]^{3 / 2}=-\left[1-\frac{1}{A}\left(\zeta+\frac{a_{1}}{2} \zeta^{2}\right)\right] \frac{\mathrm{d}}{\mathrm{d} \tau}\left\{\frac{\mathrm{d} \zeta}{\mathrm{d} \tau}\left[1-\frac{\alpha}{A}\left(1+a_{1} \zeta\right)\right]\right\}
$$

$$
C\left[\frac{1}{A}\left(1+a_{1} \zeta\right)\right]^{3 / 2}=-\left[1-\frac{1}{A}\left(\zeta+\frac{a_{1}}{2} \zeta^{2}\right)\right]\left\{\frac{\mathrm{d}^{2} \zeta}{\mathrm{d} \tau^{2}}\left[1-\frac{\alpha}{A}\left(1+a_{1} \zeta\right)\right]-\frac{a_{1} \alpha}{A}\left(\frac{\mathrm{d} \zeta}{\mathrm{d} \tau}\right)^{2}\right\}
$$

Furthermore,

$$
2 \frac{\mathrm{d}^{2} \zeta}{\mathrm{d} \tau^{2}}-\frac{2 a_{1} \alpha / A}{1-\frac{\alpha}{A}\left(1+a_{1} \zeta\right)}\left(\frac{\mathrm{d} \zeta}{\mathrm{d} \tau}\right)^{2}=-\frac{2 C\left[\frac{1}{A}\left(1+a_{1} \zeta\right)\right]^{3 / 2}}{\left[1-\frac{1}{A}\left(\zeta+\frac{1}{2} a_{1} \zeta^{2}\right)\right]\left[1-\frac{\alpha}{A}\left(1+a_{1} \zeta\right)\right]}
$$

To simplify the notations, two new functions $g_{1}(\zeta)$ and $f_{1}(\zeta)$ are introduced, they are separately defined as

$$
\begin{aligned}
& g_{1}(\zeta)=\frac{2 a_{1} \alpha / A}{1-\frac{\alpha}{A}\left(1+a_{1} \zeta\right)} \\
& f_{1}(\zeta)=\frac{2 C\left[\frac{1}{A}\left(1+a_{1} \zeta\right)\right]^{3 / 2}}{\left[1-\frac{1}{A}\left(\zeta+\frac{1}{2} a_{1} \zeta^{2}\right)\right]\left[1-\frac{\alpha}{A}\left(1+a_{1} \zeta\right)\right]}
\end{aligned}
$$


Thus, Eq. (34) is transformed as

$$
2 \frac{\mathrm{d}^{2} \zeta}{\mathrm{d} \tau^{2}}-g_{1}(\zeta)\left(\frac{\mathrm{d} \zeta}{\mathrm{d} \tau}\right)^{2}=-f_{1}(\zeta)
$$

By introducing a new variable $\omega$, which is defined as:

$$
\omega=\frac{\mathrm{d} \zeta}{\mathrm{d} \tau}
$$

Eq. (37) is finally transformed to:

$$
\frac{\mathrm{d} \omega^{2}}{\mathrm{~d} \zeta}-g_{1}(\zeta) \omega^{2}=-f_{1}(\zeta)
$$

Considering the initial condition, the solution is then given as (See the details in Appendix A )

$$
\begin{gathered}
\frac{\mathrm{d} \zeta}{\mathrm{d} \tau}=\sqrt{h_{1}(\zeta) \bar{V}^{2}+h_{1}(\zeta) \int_{0}^{\zeta}-\frac{f_{1}(x)}{h_{1}(x)} \mathrm{d} x} \\
h_{1}(\zeta)=\frac{1}{\left[1-\frac{\alpha}{A}\left(1+a_{1} \zeta\right)\right]^{2}}
\end{gathered}
$$

\subsubsection{Quadratic density gradient}

For the linear density gradient rod, $a_{1}$ and $a_{3}$ are equal to 0. Eq. (21) is therefore transformed to

$$
C\left[\frac{1}{A}\left(1+a_{2} \zeta^{2}\right)\right]^{3 / 2}=-\left[1-\frac{1}{A}\left(\zeta+\frac{a_{2}}{3} \zeta^{3}\right)\right] \frac{\mathrm{d}}{\mathrm{d} \tau}\left\{\frac{\mathrm{d} \zeta}{\mathrm{d} \tau}\left[1-\frac{\alpha}{A}\left(1+a_{2} \zeta^{2}\right)\right]\right\}
$$

Following the same process for the linear density gradient and the specific initial condition, the solution is given as

$$
\frac{\mathrm{d} \zeta}{\mathrm{d} \tau}=\sqrt{h_{2}(\zeta) \bar{V}^{2}+h_{2}(\zeta) \int_{0}^{\zeta}-\frac{f_{2}(x)}{h_{2}(x)} \mathrm{d} x}
$$

where

$$
\begin{gathered}
h_{2}(\zeta)=\frac{1}{\left[1-\frac{\alpha}{A}\left(1+a_{2} \zeta^{2}\right)\right]^{2}} \\
f_{2}(\zeta)=\frac{2 C\left[\frac{1}{A}\left(1+a_{2} \zeta^{2}\right)\right]^{3 / 2}}{\left[1-\frac{1}{A}\left(\zeta+\frac{a_{2}}{3} \zeta^{3}\right)\right]\left[1-\frac{\alpha}{A}\left(1+a_{2} \zeta^{2}\right)\right]}
\end{gathered}
$$

\subsubsection{Square root density gradient}

For the linear density gradient rod, $a_{1}$ and $a_{2}$ are equal to 0. Eq. (21) is therefore transformed to

$$
C\left[\frac{1}{A}\left(1+a_{3} \sqrt{\zeta}\right)\right]^{3 / 2}=-\left[1-\frac{1}{A}\left(\zeta+\frac{2 a_{3}}{3} \zeta^{3 / 2}\right)\right] \frac{\mathrm{d}}{\mathrm{d} \tau}\left\{\frac{\mathrm{d} \zeta}{\mathrm{d} \tau}\left[1-\frac{\alpha}{A}\left(1+a_{3} \sqrt{\zeta}\right)\right]\right\}
$$


Following the same process for the linear density gradient and the specific initial condition, the solution is given as

$$
\frac{\mathrm{d} \zeta}{\mathrm{d} \tau}=\sqrt{h_{3}(\zeta) \bar{V}^{2}+h_{3}(\zeta) \int_{0}^{\zeta}-\frac{f_{3}(x)}{h_{3}(x)} \mathrm{d} x}
$$

where

$$
\begin{gathered}
h_{3}(\zeta)=\frac{1}{\left[1-\frac{\alpha}{A}\left(1+a_{3} \sqrt{\zeta}\right)\right]^{2}} \\
f_{3}(\zeta)=\frac{2 C\left[\frac{1}{A}\left(1+a_{3} \sqrt{\zeta}\right)\right]^{3 / 2}}{\left[1-\frac{1}{A}\left(\zeta+\frac{2 a_{3}}{3} \zeta^{3 / 2}\right)\right]\left[1-\frac{\alpha}{A}\left(1+a_{3} \sqrt{\zeta}\right)\right]}
\end{gathered}
$$

\subsection{Case with a backing mass}

It is easy to add a backing mass at the rear of the rod as in the previous works (Reid and Peng, 1997; Harrigan et al., 2010; Shen et al., 2013a). A non-dimensional parameter $\gamma$ is introduced to denote the mass ratio between the rigid mass $M_{r}$ and the mass of cellular rod.

$$
\gamma=\frac{M_{r}}{\rho_{0} A_{0} l_{0}}
$$

The rigid mass parameter doesn't change the form of the governing equation. With the extra mass item, Eq. (15) becomes:

$$
\sigma_{p} A_{0}=-\left(M_{r}+M-m\right) \frac{\mathrm{d}}{\mathrm{d} t}\left\{\frac{\mathrm{d} Z}{\mathrm{~d} t}\left[1-\frac{\rho(Z)}{\rho_{d}}\right]\right\}
$$

and the specific non-dimensional form, Eq. (21), is thus:

$$
C \bar{\rho}(\zeta)^{3 / 2}=-[1+\gamma-\bar{m}(\zeta)] \frac{\mathrm{d}}{\mathrm{d} \tau}\left\{\frac{\mathrm{d} \zeta}{\mathrm{d} \tau}[1-\alpha \bar{\rho}(\zeta)]\right\}
$$

And the solutions for the different density gradients are:

- uniform density

$$
\frac{\mathrm{d} \zeta}{\mathrm{d} \tau}=\frac{\bar{V}}{1-\alpha} \sqrt{1+\frac{2 C(1-\alpha)}{\bar{V}^{2}} \ln \frac{1+\gamma-\zeta}{1+\gamma}}
$$

- Linear density gradient The form of solution Eq. (40) doesn't change, only the expression of $f_{1}(\zeta)$, Eq. (36), transforms to

$$
f_{1}(\zeta)=\frac{2 C\left[\frac{1}{A}\left(1+a_{1} \zeta\right)\right]^{3 / 2}}{\left[1+\gamma-\frac{1}{A}\left(\zeta+\frac{1}{2} a_{1} \zeta^{2}\right)\right]\left[1-\frac{\alpha}{A}\left(1+a_{1} \zeta\right)\right]}
$$

- Quadratic density gradient As the same, $f_{2}(\zeta)$ changes to

$$
f_{2}(\zeta)=\frac{2 C\left[\frac{1}{A}\left(1+a_{2} \zeta^{2}\right)\right]^{3 / 2}}{\left[1+\gamma-\frac{1}{A}\left(\zeta+\frac{a_{2}}{3} \zeta^{3}\right)\right]\left[1-\frac{\alpha}{A}\left(1+a_{2} \zeta^{2}\right)\right]}
$$


- Square root density gradient The same to the square root density gradient, $f_{3}(\zeta)$ changes to

$$
f_{3}(\zeta)=\frac{2 C\left[\frac{1}{A}\left(1+a_{3} \sqrt{\zeta}\right)\right]^{3 / 2}}{\left[1+\gamma-\frac{1}{A}\left(\zeta+\frac{2 a_{3}}{3} \zeta^{3 / 2}\right)\right]\left[1-\frac{\alpha}{A}\left(1+a_{3} \sqrt{\zeta}\right)\right]}
$$

It is noted that the calculation of dynamic crushing stresses are from the same equations but the density gradient parameters $a_{i}$ should be larger than 0 . It means that the density is growing from the striking end to the backing rigid mass end. Otherwise, two shock wave fronts will be generated as indicated by Shen et al. (2013a).

This analytical solution of governing equations provides not only the shock front speed $\frac{\mathrm{d} \zeta}{\mathrm{d} \tau}$ when the front arrives at a given position $\zeta$ but also the variation of dynamic crushing stress at this moment (shock front at the position $\zeta$ ). In particular, when the shock front speed $\frac{\mathrm{d} \zeta}{\mathrm{d} \tau}$ is reduced to zero, it means the shock front stopped at this position.

\section{Validation of theoretical analysis}

In order to validate the presented analysis and to justify the existence of the shock front propagation in density graded cellular rod, a numerical simulation with commercial FEM code (Abaqus) are performed.

The present numerical model is a 3D full FEM Abaqus model. It is a $62 \mathrm{~mm}$ long rod of a 1 $m m \times 1 \mathrm{~mm}$ square cross section. This rod is divided equally into 31 portions of $2 \mathrm{~mm}$ in length. Hourglass controlled, eight nodes linear brick, reduced integration solid element (C3D8R) with the size $0.2 \mathrm{~mm} \times 0.2 \mathrm{~mm} \times 0.2 \mathrm{~mm}$ is adopted to mesh this rod. A rigid plate is used to represent the rigid wall in the model. Surface to surface kinematic contact without friction is applied to the rigid plate and rod surfaces. The whole rod is predefined an initial velocity against the wall which is fixed.

Isotropic crushable foam model (Deshpande and Fleck, 2000) is employed with zero plastic Poisson ratio and the yield strength ratio $k$ (initial yield strength in uniaxial compression, $\sigma_{c}$, divided by initial yield strength in hydrostatic compression, $p_{c}$ ) is set to 1 . The elastic-plastic locking relationship is applied. Parameters of this isotropic crushable foam model are actually determined in the way that the simulation of a uniform cellular bar will give a good agreement with the known theoretical result. Therefore, the eventual 3D effect of the isotropic crushable foam model is not really included in our analysis of density gradient. The case of a uniform bar provides also an efficiency proof of this FEM analysis. 


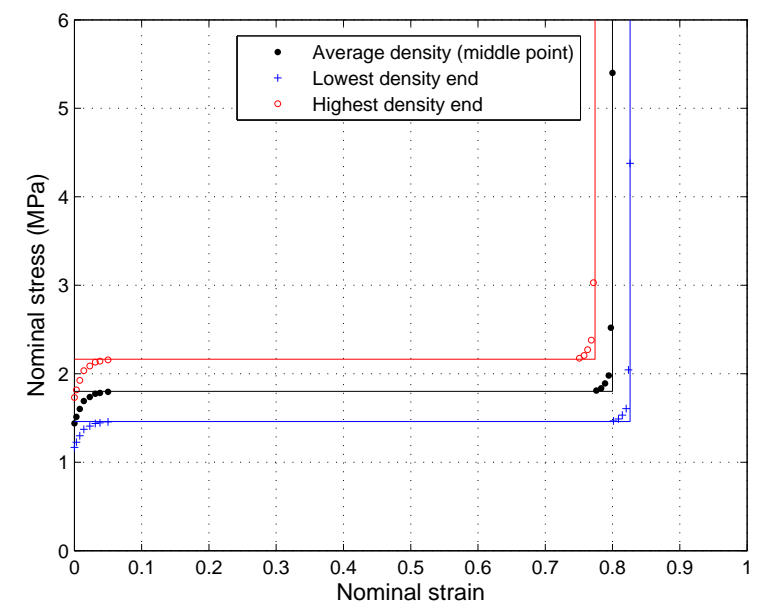

Figure 2: Typical input stress-strain curves in numerical models and their corresponding RPPL model

Afterwards, a linear density profile is applied according to the location of each portion in the longitudinal coordinate $Z$. The density in each portion varies with its central position. It is emphasized that the stress-strain relations applied to each portion are also different. The key parameters, the stress level and locking strain, are obtained with respect to the actual density of each portion. For instance, the stress level is calculated by using the Eq. (14). Fig. 2 shows the input stress-strain data in the two ends and the middle point of the rod as an example. The solid lines in Fig. 2 are the corresponding theoretical RPPL models.

Fig. 3 shows logarithmic strain longitudinal direction of rod (LE33) in cellular rod with linear density gradient $\left(a_{1}=0.3\right)$ where initial impact speed is set to be $100 \mathrm{~m} / \mathrm{s}$. A distinct shock front can be observed near the impact end and the shock front propagation speed decreases during its propagation as predicted by the theoretic analysis. It proves that the shock wave is created in a density graded cellular rod.

In addition, the dynamic crushing stresses $\sigma_{d}$ can be extracted from the FEM analysis and compared with analytical results. The case of linear density gradient $\left(a_{1}=0.3\right)$ as well as the uniform case compared with the corresponding theoretical results are given in Fig. 4, Their agreement exhibits the accuracy of theoretical formulas, especially at the propagating phase where the perturbation due to initial shock front formation is less important. 


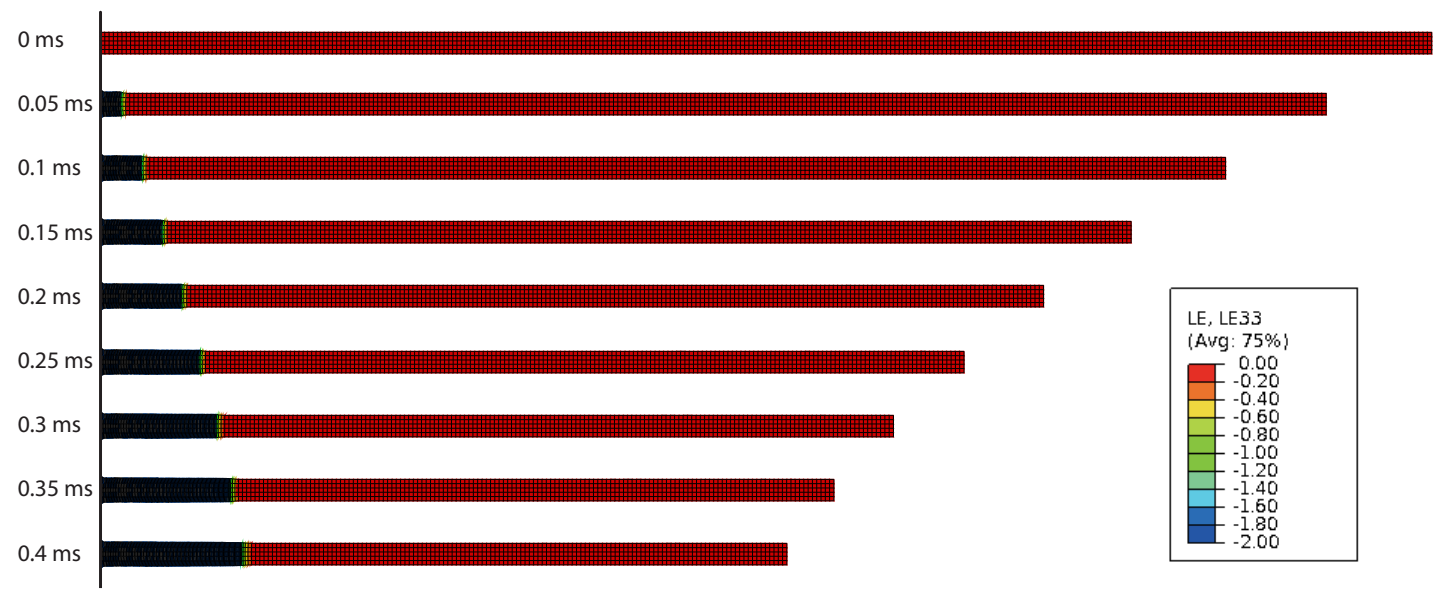

Figure 3: LE33 (logarithmic strain longitudinal direction of rod) at different time on the linear density graded cellular rod where $a_{1}=0.3$ and $V_{0}=100 \mathrm{~m} / \mathrm{s}$

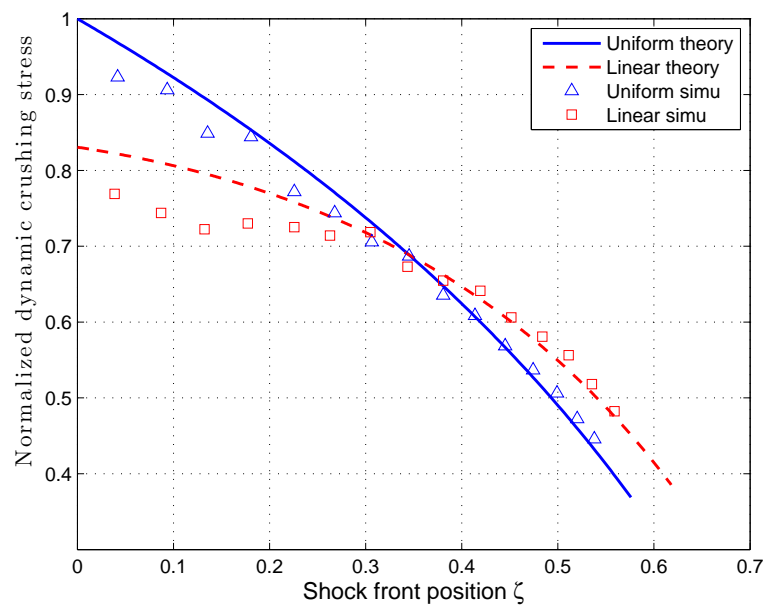

Figure 4: Comparison of dynamic crushing stress between theoretical studies (same as Fig. 7) and numerical simulations, where $a_{1}=0.3$ and $V_{0}=100 \mathrm{~m} / \mathrm{s}$ 


\section{Discussions on the effect of the gradient}

With the validation of theoretic formulas presented above, the influence of the density gradient can be now studied.

\section{1. results with a cellular rod only}

In order to illustrate the influence of density profiles, the shock front speed as well as dynamic crushing stress as a function of the position reached by this shock front are calculated for the four cases as examples. The default conditions are as follows: initial impact speed $100 \mathrm{~m} / \mathrm{s}$, density gradients $a_{1}=a_{2}=a_{3}=0.3$, and the parameter $C$ in Eq. (19) 0.0164 , if not indicated specially. Fig. 5 illustrates the default density profiles along the cellular rod, which are normalized by the uniform one. Such a gradient is rather small but it is realistic for currently produced graded foams. Of course, the sharper is the density gradient, the bigger will be its effect on the shock propagation features.

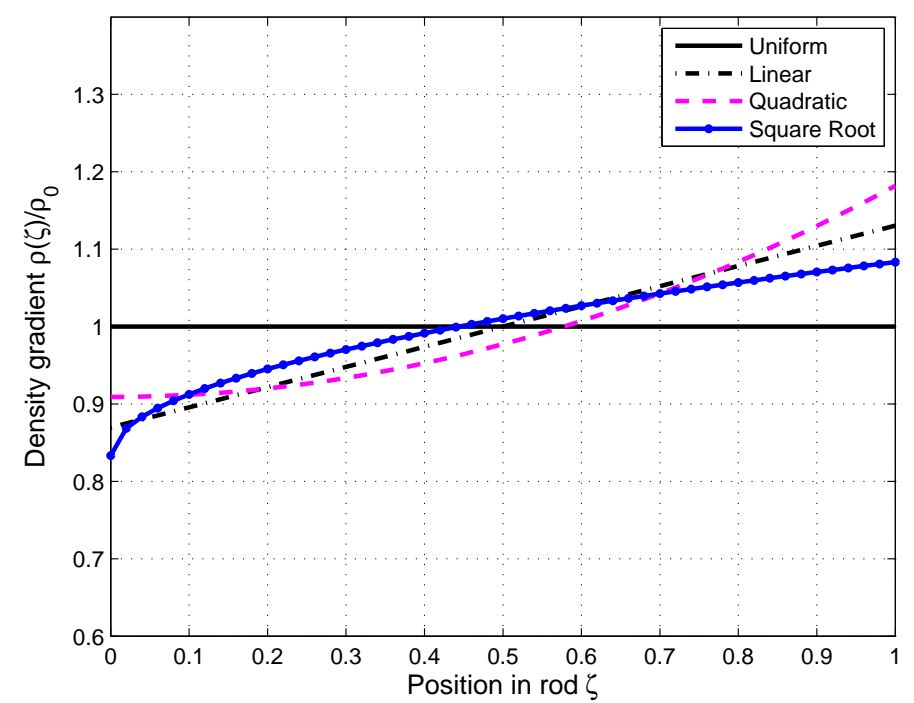

Figure 5: The typical density gradients along the cellular rod for the four different gradient functions normalized

Fig. [6] shows the non-dimensional shock front speeds of the four density profiles as a function of the shock front position. The influence of the gradients seems not to be very important because the final positions of shock front hardly changed. Nevertheless, the differences between the dynamic crushing stresses shown in Fig. 7 are more noticeable. 


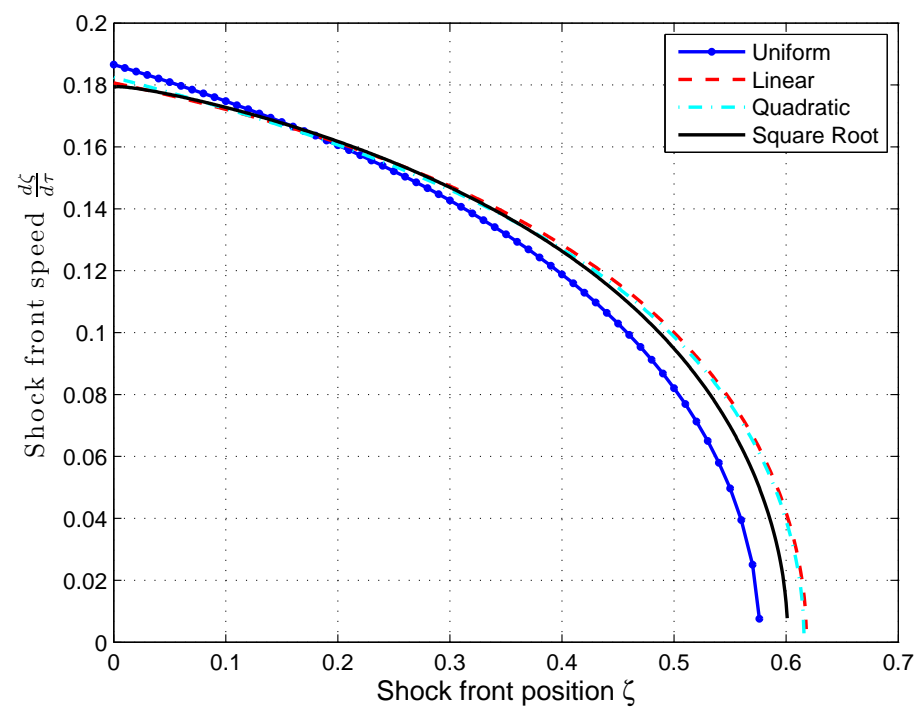

Figure 6: The four shock front velocity attenuating profiles with its position

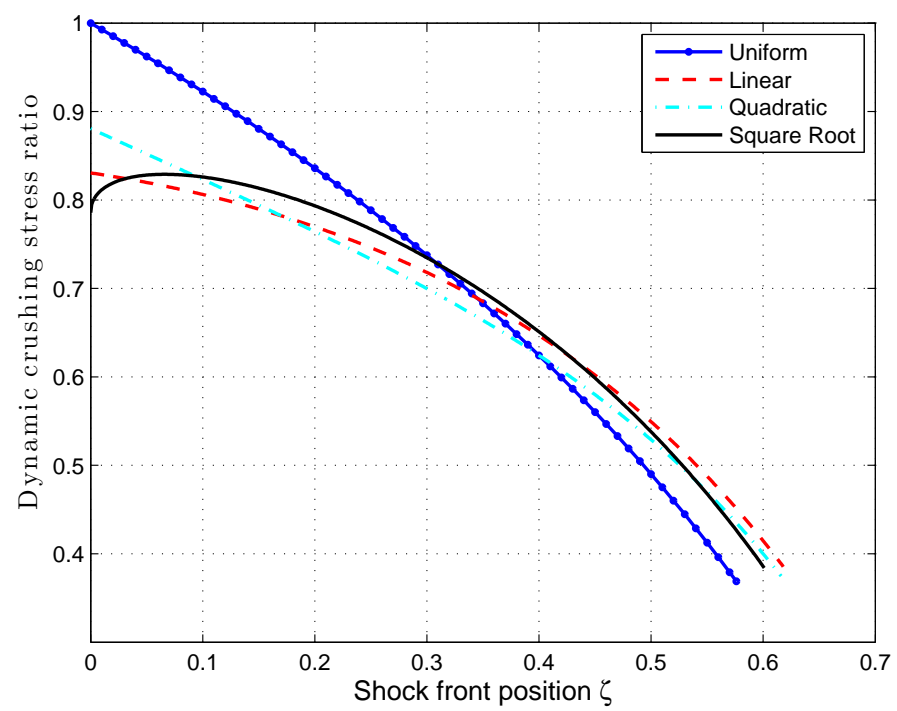

Figure 7: The four stress varying profiles with shock front position

The influence of the initial impact velocity is also studied. In Fig. 8, we can see that the gaps between the final positions of shock front are nearly unaffected by the initial impact speeds. However, the maximum dynamic stresses $\sigma_{d}$, normalized by the value obtained in the case of uniform density at the corresponding initial impact speed, are attenuated between $10 \%$ and $20 \%$ 
(see Fig. 9).

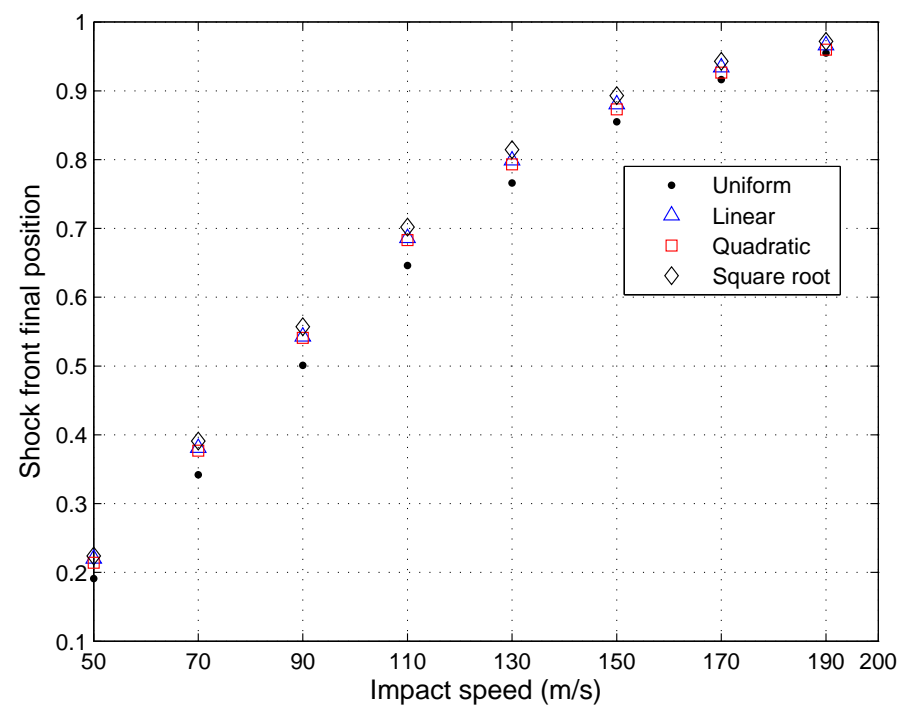

Figure 8: The final position reached by shock front for four density gradients

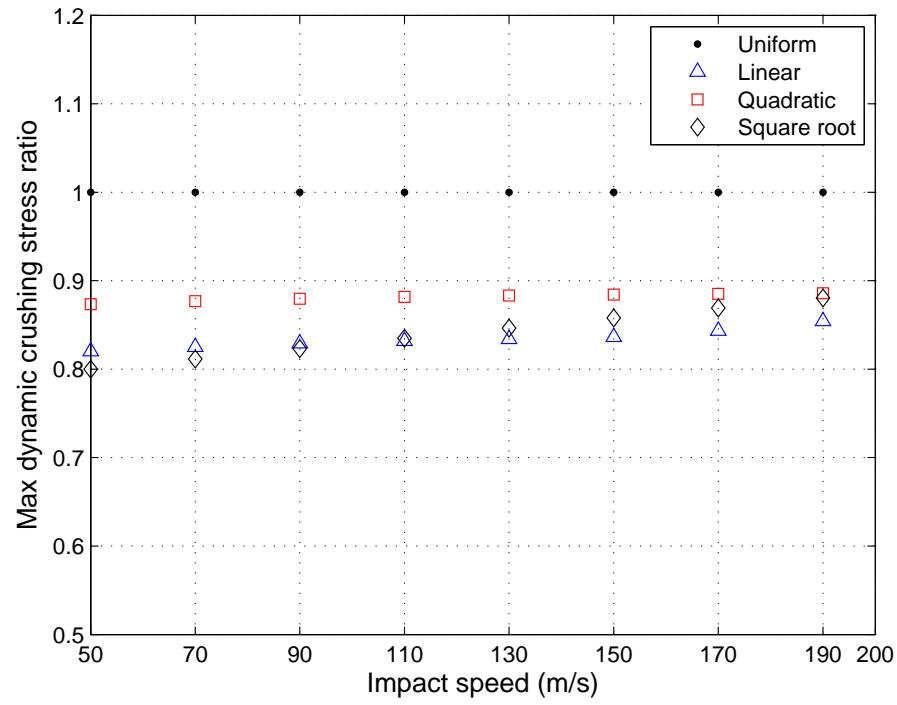

Figure 9: Max crushing stresses for different density gradients, normalized by the one of uniform density. 


\section{2. results with a backing mass}

The influence of the backing mass is also considered here. The cellular rod with a rigid mass equal to the mass of cellular rod impacts on the rigid wall at a speed $100 \mathrm{~m} / \mathrm{s}$ is taken as an example. Fig. 10 shows that the shock front speed evolution is hardly affected by the density profiles. The maximum dynamic crushing stress $\sigma_{d}$ (see Fig. 11) follows however the same type of reduction as the case of cellular rod only. Different initial impact speeds are investigated. The cellular rods are completely crushed in this case. The maximum dynamic crushing stress ratio given in Fig. 12 illustrates a stress reduction in the range of low impact velocity.

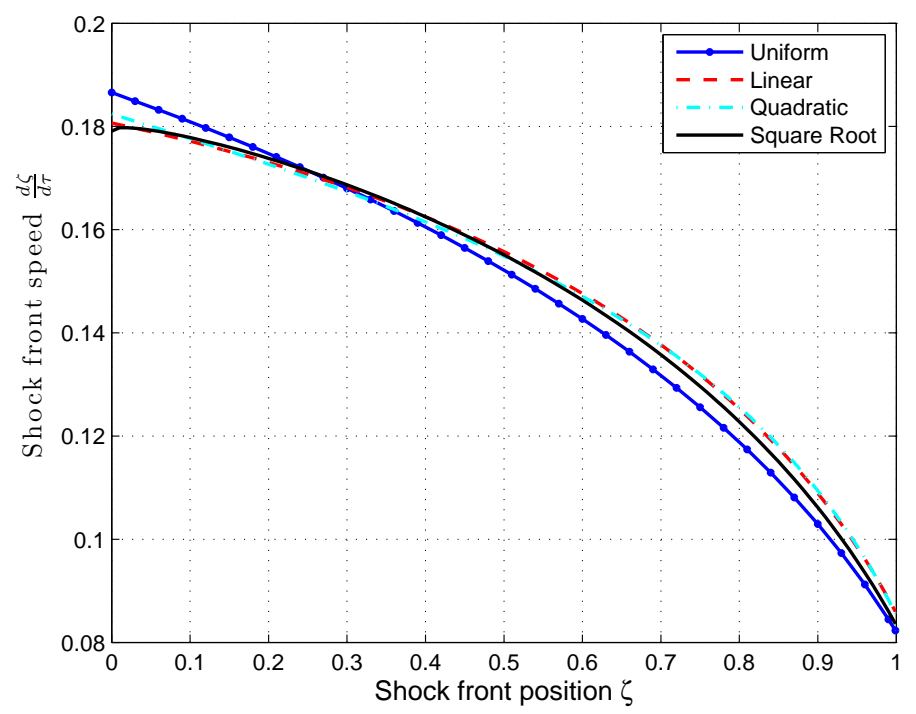

Figure 10: The shock front velocity attenuating profiles with its position, backing mass ratio $(\gamma=1)$

\subsection{Discussion on the maximum stress reduction}

From results shown above, it is quite clear that the energy absorption capacities is hardly affected by the gradient profile because the gap of final position reached by the shock front for the 4 cases is rather small. However, an interesting feature is the reduction of maximum dynamic crushing stress with the density gradient. It could be very useful for energy absorption designer because it could be a way to reduce the maximum force (to avoid the destruction of the protected device) with the same energy absorption capacity and the same weight of course.

However, it was noticed that the use of density gradient may take a risk of enlarging the maximum crushing stress at high impact velocity. A further investigation for this maximum 


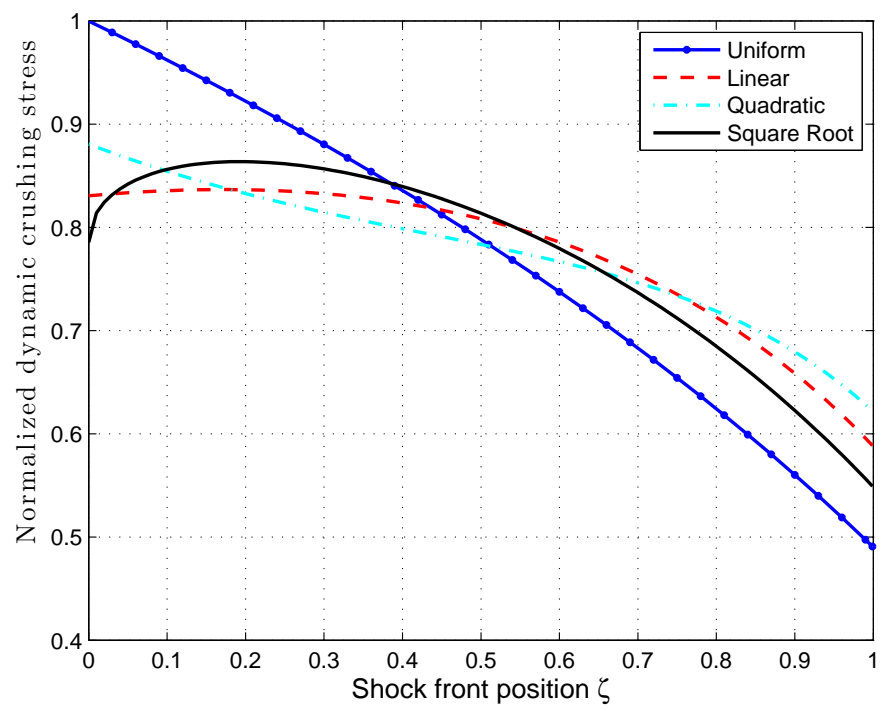

Figure 11: The stress varying profiles with shock front position, backing mass ratio $(\gamma=1)$

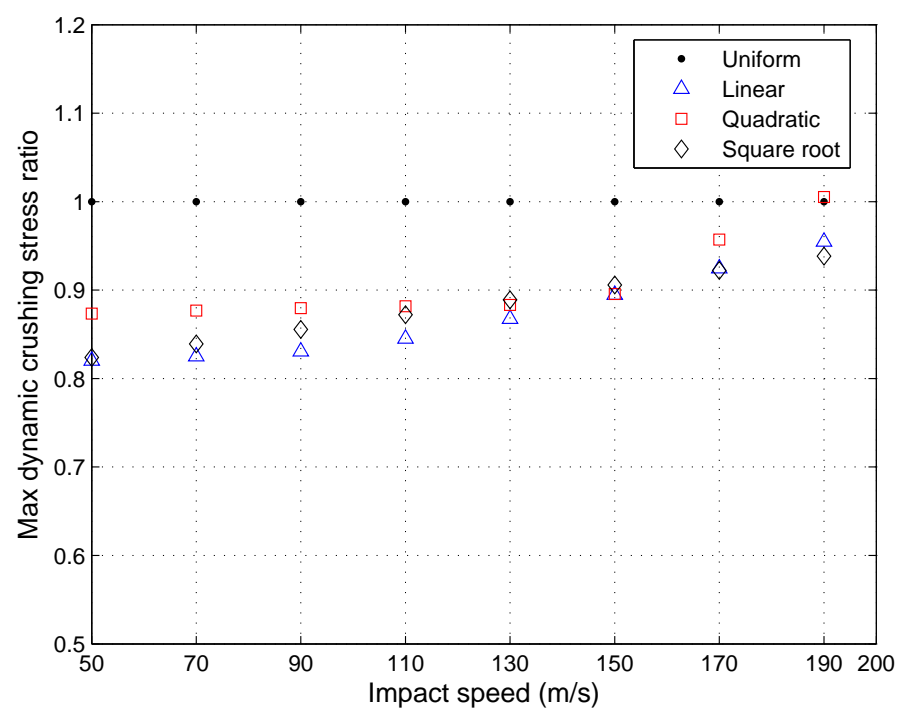

Figure 12: Max crushing stresses for different density gradients, normalized by the one of uniform density.

stress reduction is made. For the linear gradient profiles, the influence of the importance of gradient (parameter $a_{1}$ ) as well as the backing mass ratio are shown in Fig. 13, The benefit of gradient is clear for rather small value of gradient slope and the small backing mass ratio. For high gradient slope as well as important mass ratio, the dynamic crushing stress could increase 
instead of a reduction. For the quadratic gradient profiles (Fig. 14), the same conclusion can be made. While for the square root profiles (see Fig. 15), the advantage of the application of density gradient is always true in the range of consideration. Nevertheless, this advantage weakens as the backing mass ratio increases.

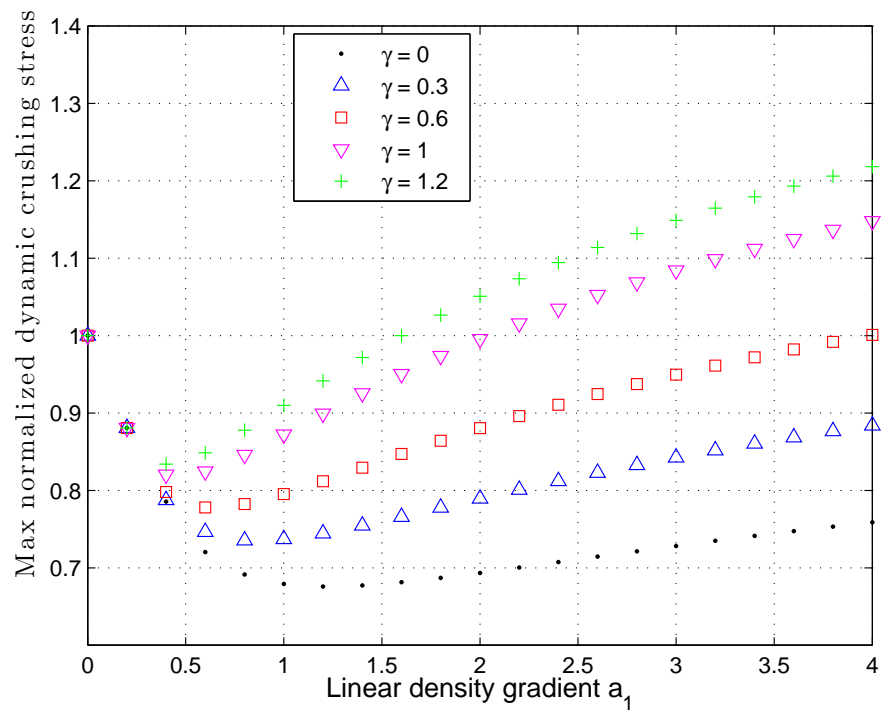

Figure 13: Max stresses in linear density gradient cellular rod with different gradients $a_{1}$, and backing mass ratio $\gamma$

\section{Summary}

This paper investigated theoretically the shock front formation/propagation in graded cellular materials for the case of more realistic (from industrial application viewpoint) property gradients derived from density gradient. On the basis of assumption of a constant locking density (instead of locking strain), the governing equations of shock front in a density graded cellular material was derived. Analytical expressions of shock front speed as well as dynamic crushing stresses for linear, quadratic and square root density profiles were obtained in the case of cellular rod with a backing mass or not.

Numerical simulations showed that shock front formed in the cellular rod and the results agreed well with the theoretical predictions. This study revealed that the density gradient had a significant influence on the maximum impact stress transmitted to impacted structure with the 


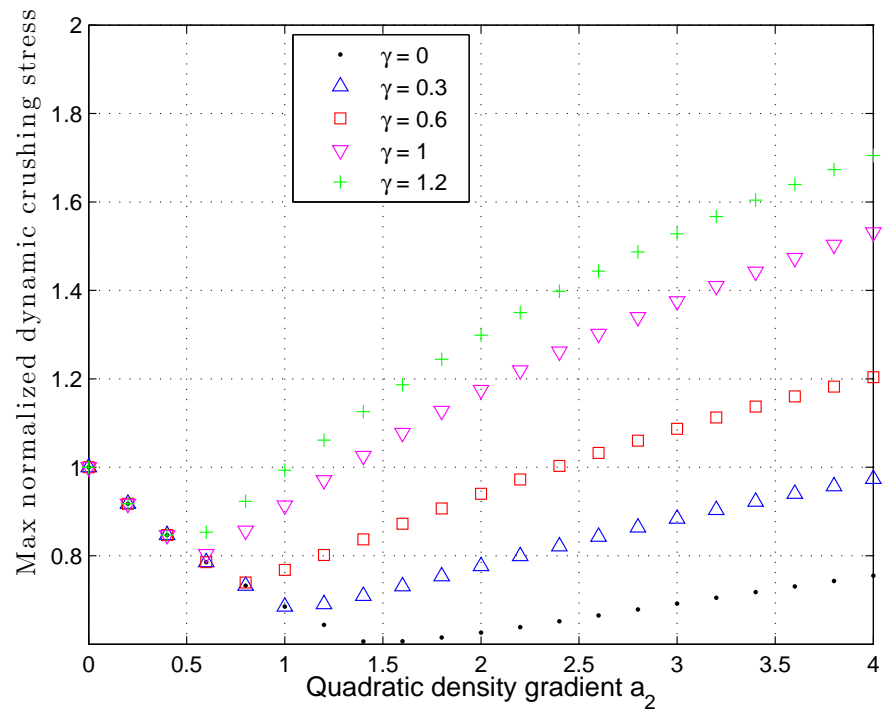

Figure 14: Max dynamic crushing stresses in quadratic density gradient cellular rod with different gradients $a_{2}$, and backing mass ratio $\gamma$



Figure 15: Max dynamic crushing stresses in square root density gradient cellular rod with different gradients $a_{3}$, and backing mass ratio $\gamma$

same weight and the same energy absorption capacity. A reduction of this maximum stress was observed when the density gradients were rather small. 


\section{Acknowledgements}

The authors acknowledge the support of China Scholarship Council (CSC) as well as NSFC funding no11228206 and no11402304.

\section{Appendix A. Solution of the differential equation}

For the first-order non-homogeneous differential equation, which is

$$
\frac{\mathrm{d} y(x)}{\mathrm{d} x}-g(x) y(x)=f(x)
$$

to obtain the solution, the homogeneous form of which is firstly resolved. It is

$$
\frac{\mathrm{d} h(x)}{\mathrm{d} x}-g(x) h(x)=0
$$

The general solution of the above equation is then

$$
h(x)=\exp ^{\int g(t) \mathrm{d} t}
$$

Thus, the general solution of the non-homogeneous equation is

$$
y(x)=K(x) h(x)
$$

Substituting the general solution into Eq. (A.1), it becomes

$$
\begin{gathered}
\frac{\mathrm{d} K(x)}{\mathrm{d} x} h(x)+K(x)\left[\frac{\mathrm{d} h(x)}{\mathrm{d} x}-g(x) h(x)\right]=f(x) \\
\frac{\mathrm{d} K(x)}{\mathrm{d} x} h(x)=f(x)
\end{gathered}
$$

As a result, $K(x)$ is solved from Eq. (A.6)

$$
K(x)=\int f(x) / h(x) \mathrm{d} x
$$

At last, the solution of the non-homogeneous different equation is thus

$$
y(x)=h(x) \int_{0}^{x} f(t) / h(t) \mathrm{d} t+C
$$

where $\mathrm{C}$ is an integral constant, which is determined by the initial condition. 


\section{References}

Ajdari, A., Nayeb-Hashemi, H., Vaziri, A., 2011. Dynamic crushing and energy absorption of regular, irregular and functionally graded cellular structures. Int. J. Solids Struct. 48 (3-4), $506-516$.

Ali, M., Qamhiyah, A., Flugrad, D., Shakoor, M., 2008. Theoretical and finite element study of a compact energy absorber. Adv. Eng. Softw. 39 (2), 95-106.

Ashby, M. F., Evans, T., Fleck, N. A., Hutchinson, J., Wadley, H., Gibson, L., 2000. Metal Foams: A Design Guide: A Design Guide. Butterworth-Heinemann.

Cui, L., Kiernan, S., Gilchrist, M. D., 2009. Designing the energy absorption capacity of functionally graded foam materials. Mat. Sci. Eng. A-Struct. 507 (1-2), 215-225.

Deshpande, V., Fleck, N., 2000. Isotropic constitutive models for metallic foams. J. Mech. Phys. Solids 48 (6), 1253-1283.

Elnasri, I., Pattofatto, S., Zhao, H., Tsitsiris, H., Hild, F., Girard, Y., 2007. Shock enhancement of cellular structures under impact loading: Part i experiments. J. Mech. Phys. Solids 55 (12), $2652-2671$.

Gibson, L., Ashby, M., 1997. cellular Solids: Structures and Properties, 2nd Edition. Cambridge University Press, UK.

Gupta, N., 2007. A functionally graded syntactic foam material for high energy absorption under compression. Mater. Lett. 61 (4-5), 979-982.

Hanssen, A., Enstock, L., Langseth, M., 2002. Close-range blast loading of aluminium foam panels. Int. J. Impact Eng. 27 (6), 593-618.

Harrigan, J., Reid, S., Yaghoubi, A. S., 2010. The correct analysis of shocks in a cellular material. Int. J. Impact Eng. 37 (8), 918-927.

Hohe, J., Hardenacke, V., Fascio, V., Girard, Y., Baumeister, J., Stöbener, K., Weise, J., Lehmhus, D., Pattofatto, S., Zeng, H., et al., 2012. Numerical and experimental design of graded cellular sandwich cores for multi-functional aerospace applications. Mater. Des. 39, $20-32$. 
Hou, B., Zhao, H., Pattofatto, S., Liu, J., Li, Y., 2012. Inertia effects on the progressive crushing of aluminium honeycombs under impact loading. Int. J. Solids Struct. 49 (19-20), 2754-2762.

Hu, L. L., Yu, T. X., 2010. Dynamic crushing strength of hexagonal honeycombs. Int. J. Impact Eng. 37 (5), 467-474.

Kammer, C., 1999. Aluminium foam. Talant Lecture 1410 - European Aluminium Association.

Lopatnikov, S. L., Gama, B. A., Haque, M. J., Krauthauser, C., Gillespie, J. W., 2004. Highvelocity plate impact of metal foams. Int. J. Impact Eng. 30 (4), 421-445.

Lopatnikov, S. L., Gama, B. A., Haque, M. J., Krauthauser, C., Gillespie, J. W., Guden, M., Hall, I. W., 2003. Dynamics of metal foam deformation during taylor cylinder-hopkinson bar impact experiment. Compos. Struct. 61 (1-2), 61-71.

Maiti, S., Gibson, L., Ashby, M., 1984. Deformation and energy absorption diagrams for cellular solids. Acta Metall. 32 (11), 1963-1975.

Nemat-Nasser, S., Kang, W., McGee, J., Guo, W.-G., Isaacs, J., 2007. Experimental investigation of energy-absorption characteristics of components of sandwich structures. Int. J. Impact Eng. 34 (6), 1119-1146.

Pattofatto, S., Elnasri, I., Zhao, H., Tsitsiris, H., Hild, F., Girard, Y., 2007. Shock enhancement of cellular structures under impact loading: Part ii analysis. J. Mech. Phys. Solids 55 (12), $2672-2686$.

Radford, D. D., Deshpande, V. S., Fleck, N. A., 2005. The use of metal foam projectiles to simulate shock loading on a structure. Int. J. Impact Eng. 31 (9), 1152-1171.

Reid, S. R., Peng, C., 1997. Dynamic uniaxial crushing of wood. Int. J. Impact Eng. 19 (5-6), $531-570$.

Said, M. R., Tan, C.-F., 2008. The response of aluminium foams under quasi-static loading. Chiang Mai J. Sci. 35, 241-249.

Shen, C., Yu, T., Lu, G., 2013a. Double shock mode in graded cellular rod under impact. Int. J. Solids Struct. 50 (1), 217-233.

Shen, C. J., Lu, G. X., Yu, T. X., 2013b. Cellular rod with varying cross-section under impact. Key Engineering Materials 535, 94-97. 
Tan, P. J., Reid, S. R., Harrigan, J. J., 2012. On the dynamic mechanical properties of open-cell metal foams - a re-assessment of the 'simple-shock theory'. Int. J. Solids Struct. 49 (19-20), $480-488$.

Tan, P. J., Reid, S. R., Harrigan, J. J., Zou, Z., Li, S., 2005. Dynamic compressive strength properties of aluminium foams. part ii - 'shock' theory and comparison with experimental data and numerical models. J. Mech. Phys. Solids 53 (10), 2206-2230.

Wang, X., Zheng, Z., Yu, J., 2013. Crashworthiness design of density-graded cellular metals. Theoretical and Applied Mechanics Letters 3 (3), 0310011-0310015.

Zeng, H. B., Pattofatto, S., Zhao, H., Girard, Y., Fascio, V., 2010. Impact behaviour of hollow sphere agglomerates with density gradient. Int. J. Mech. Sci. 52 (5), 680-688.

Zhao, H., Elnasri, I., Abdennadher, S., 2005. An experimental study on the behaviour under impact loading of metallic cellular materials. Int. J. Mech. Sci. 47 (4-5), 757-774.

Zheng, Z., Liu, Y., Yu, J., Reid, S. R., 2012. Dynamic crushing of cellular materials: Continuumbased wave models for the transitional and shock modes. Int. J. Impact Eng. 42, 66-79.

Zou, Z., Reid, S. R., Tan, P. J., Li, S., Harrigan, J. J., 2009. Dynamic crushing of honeycombs and features of shock fronts. Int. J. Impact Eng. 36 (1), 165-176. 